\title{
Publisher's Note: Spectral order statistics of Gaussian random matrices: Large deviations for trapped fermions and associated phase transitions [Phys. Rev. E 90, 040102(R) (2014)]
}

\author{
Isaac Pérez Castillo
}

(Received 30 October 2020; published 6 November 2020)

DOI: 10.1103/PhysRevE.102.059902

This paper was published online on 30 October 2014 with an improperly formatted author affiliation. Isaac Pérez Castillo's affiliation should read "Departamento de Sistemas Complejos, Instituto de Física, Universidad Nacional Autónoma de México, México D.F. C.P. 04510, México.” The paper has been corrected as of 3 November 2020. The author affiliation is incorrect in the printed version of the journal. 